\title{
DOES EXTERNALISM ENTAIL THE ANOMALISM OF THE MENTAL?
}

\author{
By Nigholas Shea
}

In 'Mental Events' Donald Davidson argued for the anomalism of the mental on the basis of the operation of incompatible constitutive principles in the mental and physical domains. Many years later, he has suggested that externalism provides further support for the anomalism of the mental. I examine the basis for that claim. The answer to the question in the title will be a qualified 'Yes'. That is an important result in the metaphysics of mind and an interesting consequence of externalism.

\section{BACKGROUND TO THE ARGUMENT}

Davidson's claim that externalism about the contents of thoughts supports the anomalousness of the mental is found in his paper 'Could There Be a Science of Rationality?':

There are several reasons for the irreducibility of the mental to the physical....

Another reason, perhaps easier to grasp, lies in the irreducibly causal character of mental concepts. Let me give a non-mental example first. The state of being sunburnt is necessarily a state caused by the action of the sun. No completed physics would make use of the concept of sunburn, not only because part of the explanation is already built into the characterization of the state, but also because two states of the skin could be in every intrinsic way identical, and yet one be a case of sunburn and the other not.

The propositional attitudes, the semantics of spoken words, and behaviour as we normally understand it, are all like this. The reason, both in the case of the attitudes and in the case of semantics, is the same: what our words mean, and what our thoughts are about, is partly determined by the history of their acquisition. The truthconditions of my sentence 'The moon is gibbous', or of my belief that the moon is gibbous, depend in part on the causal history of my relations to the moon. But it could happen that two people were in relevantly similar physical states (defined just in terms of what is within the skin), and yet one could be speaking or thinking of our moon, and the other not. 
... it is only if mental properties are supervenient on the physical properties of the agent that there can be any hope of identifying the mental properties with physical properties, or of finding lawlike connections between the two. If mental properties are supervenient not only on the physical properties of the agent but in addition on the physical properties of the world outside the agent, there can be no hope of discovering laws that predict and explain behaviour solely on the basis of intrinsic features of agents.

... What does follow is that psychology cannot be reduced to physics, nor to any other of the natural sciences. ${ }^{1}$

My project is to see whether these general considerations can be converted into a detailed argument. The idea will be to find premises which are reasonably supported by these considerations and which support a valid argument to anomalousness. In the end I argue that only a qualified conclusion is entailed. To anticipate, the argument only applies (I) in the physical to mental direction; (2) to laws applying to relatively proximal entities; and (3) to laws relating the mental to basic physics - the argument does not speak to the existence of strict laws relating the mental to those special sciences which also deal in extrinsic predicates.

The underlying idea is that intrinsic physical properties and extrinsic mental properties are the wrong kinds of things to fit together in strict causal laws. Basic physics is assumed to be a system of strict causal laws which treat exclusively of intrinsic predicates. That premise may be controversial, but my purpose is to see whether the conclusion follows from Davidson's assumptions.

The distinction between intrinsic and extrinsic properties is notoriously difficult to draw. ${ }^{2}$ I shall rely upon a relatively rough characterization: intrinsic properties of an object, event or other entity depend only upon how things are with that entity itself. To make an argument for anomalousness based on the incompatibility of intrinsic and extrinsic predicates, it must be assumed that some principled distinction can ultimately be drawn between the two.

I shall assess the argument without discussing the nature of causal laws. All I need to assume is that a strict law must be at least an exceptionless true generalization, and thus that any generalization which can be shown to have exceptions cannot qualify as a strict law.

I take the anomalousness of the mental to be the thesis expressed in Davidson's 'Mental Events' and at the start of the quotation above, namely,

${ }^{1}$ Davidson, 'Could There Be a Science of Rationality?', International Fournal of Philosophical Studies, 3 (I995), pp. I-I6, at pp. $4^{-6 .}$

2 See D. Lewis and R. Langton, 'Defining "Intrinsic", Philosophy and Phenomenological Research, 58 (1998), pp. 333-45, and 'Marshall and Parsons on "Intrinsic"', Philosophy and Phenomenological Research, 63 (200I), pp. 353-6; Lewis, 'Redefining "Intrinsic", Philosophy and Phenomenological Research, 63 (200I), pp. 38I-98. 
the idea that there are no strict laws correlating phenomena classified as mental with phenomena classified as physical. ${ }^{3}$ Sometimes it appears that Davidson means something stronger than this by 'anomalousness'. My conclusion will be that the argument from externalism does not entail anomalousness in any stronger sense than as the mere absence of strict laws (see §III below). In particular, the argument is compatible with the existence of ceteris paribus laws. Nor is the form of argument distinctive of the mental domain. Many special sciences arguably employ extrinsic predicates, so the same form of argument implies the absence of strict laws between these sciences and basic physics.

My formulation of the argument is agnostic about the metaphysical status of properties. Davidsonian nominalism can be maintained by reading ' $x$ has mental property $P$ ' as 'Mental predicate " $P$ " is true of $x$ '. Similarly, 'mental particular' can be read as 'particular describable by mental predicates'. Within this picture, two events are causally related in extension, regardless of how they are described. The nomological principle of causality ensures that any two events related as cause and effect will fall under some strict law ('Mental Acts', p. 208). I also follow Davidson in assuming the supervenience of mental properties on physical properties. Supervenience raises some further issues which I explore in §II.4 below.

Furthermore, I shall leave aside the issue of whether the argument should be stated in terms of events, states, processes or other phenomena. Famously, Davidson in 'Mental Acts' dealt in events; but his arguments can equally be applied to states or other particulars. In 'Could There Be a Science of Rationality?' he seems to adopt the wider stance in talking of mental and physical 'entities'.

Finally, I adopt a standard interpretation of the externalist thoughtexperiments as supporting a modal externalism, which is the negation of internalism, formulated as follows: for any possible worlds $w_{1}$ and $w_{2}$ and individuals $x$ and $y$, if $x$ has mental property $M$ in $w_{1}$, and $y$ is an intrinsic duplicate in $w_{2}$ of $x$, then $y$ has $M$ in $w_{2}$. I shall assume that the thoughtexperiments do support this modal claim, even if on their face they seem only to lead to constitutive externalism.

\section{Structure}

The remainder of the paper is in two parts. In §II the argument is worked out in detail and the qualified conclusion is reached. In the process I examine how the conclusion squares with the supervenience of the mental

\footnotetext{
${ }^{3}$ See Davidson, 'Mental Events', repr. in his Essays on Actions and Events (Oxford: Clarendon Press, 1980), at p. 223.
} 
on the physical. In $\S I I I$ the argument is clarified in two respects: (a) the sense in which the laws of physics must be assumed to be strict; and (b) whether the argument supports anomalousness in Davidson's sense.

\section{THE ARGUMENT AND ITS LIMITATIONS}

\section{Premises}

The general idea in 'Could There Be a Science of Rationality?' is that a basic physics of strict laws deals in intrinsic properties of the entities in question, whereas externalism can individuate as different two entities which are intrinsic duplicates. Crudely, extrinsic properties are the wrong sort of thing for incorporation into basic physics, and hence psychophysical laws cannot be strict. This idea can be made precise in two key premises:

Pr. Basic physics provides a closed and complete system of strict laws couched in intrinsic predicates applying to everything that there is ${ }^{4}$

$\mathrm{P}_{2}$. [from externalism] There can be events which are in every way intrinsically indiscernible, ${ }^{5}$ but which satisfy what I shall call 'contrary extrinsic predicates': that is to say, some extrinsic predicate which applies to one of the events does not apply to the other.

\section{II.I. Physical to mental causation}

The argument is best illustrated in the case of laws covering causation from a physical cause to a mental effect. The other cases - the mental to physical direction (where the argument fails), and the synchronic case - will be considered later. Suppose a pair of intrinsically indiscernible events $e_{1}$ and $e_{2}$ satisfy contrary extrinsic predicates $M\left(e_{1}\right), \neg M\left(e_{2}\right)$, as admitted by $\left(\mathrm{P}_{2}\right)$. By $(\mathrm{PI})$, both will have physical causes (causes describable in physical intrinsic predicates). The idea is that these causes are intrinsically indiscernible. However, that requires a further assumption:

Ar. Pairs of intrinsic predicates which satisfy contrary extrinsic predicates may have intrinsically indiscernible proximate causes.

That assumption is also reasonable given the considerations relied upon by Davidson, since externalism is supported by some thought-experiments where not only the thinkers, but also their spatially and temporally local

${ }^{4} \mathrm{I}$ assume for convenience that the laws of physics are deterministic. The same argument can be re-run on probabilistic basic laws, provided the laws of physics strictly determine the objective chances of various outcomes. The required modifications are outlined in §III below.

5 'Indiscernible' is used without epistemic import as a shorthand for entities which although numerically different are intrinsically the same in every respect. 
environments are intrinsically physically indiscernible in two cases, yet they differ in mental properties. ${ }^{6}$ Taking up Davidson's example, Oscar and his twin Toscar may be on intrinsically indiscernible planets having their retinas stimulated in intrinsically indiscernible ways by intrinsically indiscernible light passing through the atmosphere and into their eyes, yet Oscar may be thinking about the moon when Toscar is not. So $(\mathrm{AI})$ seems warranted.

To continue the argument employing (AI), suppose $c_{1}$ and $c_{2}$ are intrinsically indiscernible and cause $e_{1}$ and $e_{2}$ respectively, and (for reductio) that some strict law relates $P$, some intrinsic characterization of $c_{1}$, and $M$ :

Li. $P \rightarrow M$.

That is, all events that satisfy $P$ cause events that satisfy $M$. Since $c_{2}$ is intrinsically indiscernible from $c_{1}, P$ must also apply to $c_{2}$. But if $(\mathrm{LI})$ represents a strict law, then since $c_{2}$ satisfies $P, e_{2}$ must satisfy $M$. But that contradicts the assumption $\left(\mathrm{P}_{2}\right)$ from externalism that $\neg M\left(e_{2}\right)$. So there can be no such strict law.

The argument so far has established that there can be no strict law relating any intrinsic description of a cause to the extrinsic description of an effect to which contrary extrinsic predicates may apply. However, this only covers the case of intrinsically indiscernible pairs of causes and effects, in assumption $(\mathrm{AI})$. It does not show that no strict law of causation treats of the extrinsic predicate $M$ and intrinsic properties of some cause.

The generality of the argument depends upon how far assumption $\left(\mathrm{A}_{\mathrm{I}}\right)$ can be extended. Since some externalist thought-experiments justify the assumption that two worlds can be alike in all their intrinsic properties for a considerable time, and yet differ in their mental properties, the conclusion can be reached that no strict laws relating intrinsic physical predicates to mental predicates can apply to any of the causal relations in such a period. The intrinsic physical properties dealt with by both scientific and folk explanation in describing causation usually draw upon relatively proximal causes, in the sense that the causes are more proximal than the causal or historical factors relied upon by externalism to distinguish intrinsically indiscernible mental entities. So the conclusion rules out strict laws relating the relatively proximal causes and effects usually considered by science and the folk. The argument does not exclude the existence of a strict law relating a mental description of an effect to the physical properties of some distal cause (namely, that upon which the extrinsic characterization depends). We would expect there to be at least some exceptionless generalizations employing such distal causes, for example, the claim that all sunburn is

${ }^{6}$ See, e.g., Davidson, 'Knowing One’s Own Mind' (1987), repr. in Q. Cassam (ed.), SelfKnowledge (Oxford UP, I994), pp. 43-64. 
caused by a sun. ${ }^{7}$ Exceptionless generalizations of this sort, which reach back beyond the ground for the externalist difference, are not excluded.

The strength of the argument depends upon the source of the externalism. Some causal theories of reference assume that thought contents depend upon the nature of the entity at the end of some current causal (usually perceptual) chain. But they need not reach back into the past. (Other causal theories reach further back, e.g., to an initial baptism.) So the argument will only rule out strict laws regarding relatively proximal entities (like streams of photons or patterns of retinal stimulation). Other externalist theories go back further, making the argument correspondingly stronger. For example, teleosemantics implies that thinkers can share all their current intrinsic properties and all their recent history, and yet have thoughts with different contents, because of differences in the evolutionary functions of their representational systems. If that is correct, then the argument will have a wide application, excluding strict laws across a broad swathe of space and time.

My conclusion, in short, is that the argument from externalism to the absence of strict laws of physical to mental causation is valid in an important class of cases, namely, those from intrinsic descriptions of physical events to spatially and temporally local mental events. Since science is largely concerned with establishing laws in exactly this domain, the lack of full generality does little to undermine the power of the result. Ex hypothesi the argument only applies to mental entities that are extrinsically individuated. Externalists can hold the view that some mental properties are extrinsically individuated while others are intrinsically individuated (e.g., narrow as well as broad contents). The argument of course only applies to those mental properties which one's brand of externalism says are extrinsically individuated. As to soundness, the assumptions needed to make the argument valid, while controversial, are founded in the considerations offered by Davidson in the passage quoted at the outset.

\section{II.2. Mental to physical causation}

The same style of argument does not work for mental to physical causation. Suppose a pair of physically indiscernible causes $c_{1}$ and $c_{2}$ are described by contrary extrinsic predicates $M$ and $\neg M$, relying on $\left(\mathrm{P}_{2}\right)$. By $\left(\mathrm{P}_{\mathrm{I}}\right)$, they will cause physically indiscernible chains of effects. No contradiction is produced by supposing that there is some strict law relating $M$ to some physical description $P$ of one of these effects $e_{1}$. $e_{1}$ has a physically indiscernible

7 One reading of this generalization is that all sunburn must be caused by our sun. Such generalizations involving individuals are widely thought not to be candidates for natural laws. So I shall leave aside the issue of whether externalism individuates entities as different depending upon their relations to different but intrinsically indiscernible individuals. Accordingly, I take 'sun' in the example to mean anything qualitatively like our sun. 
counterpart $e_{2}$ such that $c_{2}$ caused $e_{2}$. But that is compatible with $\neg M\left(c_{2}\right)$. A strict law that all $M$-events cause $P$-events is compatible with non- $M$ events also causing $P$-events.

Thus the direction of causation produces an asymmetry in the argument's applicability. A further assumption could be added to the effect that strict laws are time-reversible, so that the law $M \rightarrow Q$ would imply that all $Q$-type events are caused by $M$-type events. But that controversial assumption does not follow from Davidson's framework.

\section{II.3. Synchronic lawes: physical to mental}

The argument-form rules out synchronic laws from the physical to the mental in the same way as it applies to diachronic laws. Suppose a pair of intrinsically indiscernible events $a_{1}$ and $a_{2}$ satisfy contrary extrinsic predicates $M\left(a_{1}\right)$ and $\neg M\left(a_{2}\right)$, by $\left(\mathrm{P}_{2}\right)$. The existence of a strict law, couched in terms of any intrinsic physical properties $P$ of $a_{1}$ such that $P \rightarrow M$, is blocked by the fact that $P$ must also apply to $a_{2}$, but $M$ does not. This result is no surprise, since externalism was formulated as the claim that the intrinsic physical properties of a mental entity do not fix its mental properties. The additional assumption (AI) concerning indiscernible proximal causes is not needed in the synchronic case.

\section{II.4. Compatibility with the supervenience of the mental on the physical}

An obvious worry arises: if externalism about the individuation of mental entities blocks the existence of strict synchronic laws from the physical to the mental, does it not contradict the supervenience of mental properties on physical properties?8 After all, the idea of supervenience is that once all the physical properties are fixed, all the properties are fixed, including the mental properties.

This issue merits careful examination, since it goes to the heart of the tension between externalism and strict laws. Davidson states his supervenience claim as follows:

I. A mental predicate $M$ is supervenient on a set of physical predicates $P$ iff $M$ does not distinguish any entities that cannot be distinguished by $P{ }^{9}$

A more precise formulation, intended to be faithful to (I), allows some distinctions to be drawn out:

${ }^{8}$ In his 'Lewis, David: Reduction of Mind', in S. Guttenplan (ed.), A Companion to the Philosophy of Mind (Oxford: Blackwell, I994), pp. 412-31, Lewis argues that supervenience entails reduction: see pp. 4I2-2I.

${ }^{9}$ Davidson, 'Thinking Causes', in J. Heil and A. Mele (eds), Mental Causation (Oxford: Clarendon Press, 1994), pp. 3-17. 
2. A class of mental properties $M$ supervenes on a class of physical properties $P$ iff (by definition)

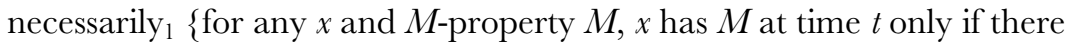
exists a $P$-property $P$ (physical base property) such that $x$ has $P$ at $t$, and necessarily $_{2}$ (anything that has $P$ at a time has $M$ at that time) $\} .{ }^{10}$

Definition (2) admits of many different strengths of supervenience claim, depending upon the strengths attributed to the two necessity operators (each of the operators may independently be either metaphysical or nomological necessity, or logical necessity, if that is thought to be different from metaphysical necessity), and also depending upon the domains from which the two classes of properties are drawn. If the physical properties are permitted to range over $x$ 's extrinsic as well as intrinsic properties, then a merely global supervenience claim is made. Alternatively, this can be seen as taking the $M$-properties of $x$ to supervene on all the physical properties of the whole world: hence the term 'global supervenience'. (The two formulations are equivalent on the assumption that any property of the world can be reformulated as some extrinsic, possibly highly relational, property of any given particular $x$.) If the class of physical properties $P$ is restricted to intrinsic properties of $x$, then a narrow supervenience claim results. Along another dimension of variation, (2) as stated is a claim of 'strong' supervenience. Dropping 'necessarily, results in 'weak' supervenience.

Although externalism is not consistent with any narrow supervenience claim, it is prima facie consistent with a merely global supervenience claim. Externalism is the claim that the intrinsic physical properties of an entity do not fix its mental properties. By contrast, global supervenience is the claim that all the physical properties (intrinsic and extrinsic) of an entity (or, as before, all the intrinsic properties of the world) do fix its mental properties.

A criticism which has often been made of Davidson's position is that even global supervenience may bring with it the existence of some physicalpsychological laws, as follows. ${ }^{11}$ If $x$ has mental property $M$ and $x$ 's mental properties are fixed by some class $Q$ of (wide) physical properties, then necessarily there is a $Q$-property $P$ such that $x$ has $P$, and necessarily anything which has $P$ has $M$. Is that not a strict law?

This objection is actually irrelevant to the argument I am considering here: the existence of laws from extrinsic physical properties to mental properties would be compatible with the absence of strict laws from intrinsic

${ }^{10}$ Taken from J. Kim, Mind in a Physical World (MIT Press, I998), p. 9, and S. Sturgeon, 'Humean Chance: Five Questions for David Lewis', Erkenntnis, 49 (I998), pp. 32 I-35.

${ }^{11}$ See, e.g., Kim, 'Supervenience and Nomological Incommensurables', American Philosophical Quarterly, I5 (1978), pp. I49-56, and 'Concepts and Supervenience', Philosophy and Phenomenological Research, 45 (1984), pp. I53-76; J. Dupré, The Disorder of Things (Harvard UP, 1993). 
physical properties to mental properties. ${ }^{12}$ However, the existence of strict laws treating of extrinsic physical properties might suggest that I was looking in the wrong place for strict laws by focusing only on intrinsic physical properties. The philosophical position is more satisfactory if supervenience does not entail the existence of strict (wide) laws. I would argue that it does not, but to make that case is beyond the scope of this paper. Two short observations will suffice. First, supervenience only implies that the mental and physical properties are necessarily co-extensive, which, on many views, is not a sufficient condition for those properties to be related by natural law. Secondly, a weak supervenience claim does not entail the necessary coextensiveness of the physical and mental properties, but just that they must be co-extensive in the actual world. And Kim's counter-argument that weak supervenience entails strong supervenience relies on the class of properties being closed under negation and disjunction. ${ }^{13}$ Those sympathetic with the view that necessary co-extensiveness is not sufficient for natural law are also likely to doubt that the class of natural properties is closed under negation, disjunction, and possibly conjunction.

In summary, there are tenable positions in which the absence of strict physical to mental synchronic laws is compatible with the supervenience (global and/or weak) of the mental on the physical.

\section{II.5. Synchronic lawes: mental to physical}

Just as the argument-form does not work with mental to physical causation, it also does not apply to synchronic laws from mental properties of an entity to physical properties of the same entity. A law that all $M$-events must have physical property $P$ is compatible with some (intrinsically indiscernible) non- $M$-event also having physical property $P$.

A different consideration, namely, multiple realization, might block the existence of such strict laws. Multiple realizability is close to the denial of strict mental to physical laws (reductions). However, on some views a mental property reduces to the disjunction of its realizers. That conclusion can be resisted on the basis of the general considerations about properties and laws discussed in the previous section, the idea that not all disjunctions of physical properties are themselves physical properties suitable to figure in laws. For example, Kim argues (Mind in a Physical World, ch. 4) that when a

12 For a version of the argument that global supervenience does not entail reductionism see B. Petrie, 'Global Supervenience and Reduction', Philosophy and Phenomenological Research, 48 (1987), pp. I19-30.

13 See Kim, 'Supervenience and Nomological Incommensurables' and 'Concepts and Supervenience'; J. Bacon, 'Supervenience, Necessary Co-extension, and Reducibility', Philosophical Studies, 49 (1986), pp. I63-76; J. Van Cleve, 'Supervenience and Closure', Philosophical Studies, $5^{8}$ (1990), pp. 225-38. 
functionally defined concept is multiply realized, there is no property which corresponds to the disjunction of the physical realizations. Rather, laws operate separately on each of the physical disjuncts, and this heterogeneous collection of realizers may achieve conceptual unity as a result of the fact that they all act as realizers for the same functionally defined state. If this is right, multiple realization of mental properties would imply the absence of any strict synchronous laws from the mental properties of any entity to its physical properties. But the same conclusion cannot be founded on the externalist considerations upon which Davidson was relying.

\section{II.6. Mental to mental causation}

The form of argument I am considering relies upon a mismatch between the intrinsic properties of the physical domain and the extrinsic properties of the mental domain. Thus it cannot be applied to laws interrelating mental predicates.

\section{COMMENTS ON THE ARGUMENT}

\section{(a) Strictness}

It would be of little interest to assess the validity of an argument from externalism to anomalousness if the premises were obviously false. An immediate objection to assumption $\left(\mathrm{P}_{\mathrm{I}}\right)$ is that quantum mechanics makes it likely that the basic laws of physics are probabilistic. However, with slight modification, the same argument works in the same cases to show that there cannot be exceptionless physical to mental laws of objective chance. The premises can be reformulated as follows:

PI*. Basic physics provides a closed and complete system of laws couched in intrinsic predicates applying to everything that there is, which determine without exception the probabilities of various outcomes characterized in terms of their intrinsic physical properties

$\mathrm{P}_{2} *$. [from externalism] There can be events which are in every way intrinsically indiscernible, but which are such that some extrinsic predicate which applies to one of the events could not apply to the other, which I shall again call contrary extrinsic predicates, broadening the terminology used in $\left(\mathrm{P}_{2}\right) .{ }^{14}$

Again the argument applies to all pairs of proximate causes and effects that are indiscernible (i.e., to which assumption $\left(\mathrm{AI}_{\mathrm{I}}\right)$ applies), but where the effects satisfy contrary extrinsic predicates, as follows:

14 The idea is that in intrinsically indiscernible worlds, whilst Oscar's thought is about water, Toscar's could not be, since he has the wrong history or context. 


$$
\begin{aligned}
& c_{1} \rightarrow e_{1} \\
& c_{2} \rightarrow e_{2} \\
& M\left(e_{1}\right), \neg M\left(e_{2}\right) .
\end{aligned}
$$

For example, while event $e_{1}$ is Oscar's thought about water, event $e_{2}$ is not and could not be about water, since Toscar lives in the wrong sort of environment for water thoughts. Now suppose for reductio that some exceptionless law fixes the objective chance of an $M$-type event based on the intrinsic physical properties $P$ of the cause:

LI*. $P \rightarrow M$ with objective chance $q$, where o $<q \leqslant \mathrm{I}, P\left(c_{1}\right)$

Since $c_{2}$ on twin earth is intrinsically indiscernible from $c_{1}$ on earth, $P$ applies to $c_{2}$, and thus law $\left(\mathrm{LI}^{*}\right)$ attributes a non-zero probability $q$ to an outcome which has mental property $M$. But that contradicts $\left(\mathrm{P}_{2} *\right)$ - no event on twin earth can have property $M$. Hence there can be no such strict law.

Thus the same conclusion, mutatis mutandis, is reached as above, where it was assumed that the laws of physics were strict in both the senses, exceptionless and non-probabilistic. That is, on reasonable assumptions, the argument from externalism does establish the impossibility of exceptionless laws of objective chance covering physical to mental causation in an important class of cases, namely, those from intrinsic descriptions of physical events to spatially and temporally proximal mental events.

\section{(b) Anomalous, but not distinctive of the mental}

The form of argument I have been considering establishes that physicalmental laws must have exceptions (in the class of cases to which the argument applies). However, the style of argument can be used in considering laws relating basic physics to any other field which employs extrinsic predicates, including biology and some other 'special sciences'. In short, the argument establishes anomalousness qua extrinsic, as in the other special sciences, not anomalousness qua mental. If it is suggested by Davidson or others that the anomalousness of the mental is distinctive of the mental realm, then the argument I have been considering does not establish anomalousness in that stronger sense. In particular, it is consistent with the argument that there should be ceteris paribus laws relating the mental and physical domains.

However, this does not make the argument from externalism valueless. It still demonstrates that the mental domain is irreducible to the physical, 'irreducible' in a sense sufficiently strong to ensure that mental properties are explanatorily ineliminable. Thus it answers one species of mind-body problem: how there can be room in the world for mental properties when 
everything that there is forms part of a closed and complete system characterizing every particular in terms of physical properties. To put this another way, it is the problem of how there can be a separate system of mental predicates which apply only to some entities, when there is a system of physical predicates which characterize every entity in a closed and complete system. Why are the mental predicates not just a notational variant of the physical predicates? In the absence of strict laws relating the mental and the physical there is room for at least two schemes for classifying phenomena, in terms of physical and mental predicates respectively. Since mental predicates are not reducible to physical ones, they may be an alternative means of classification which has independent explanatory validity. The block on reducibility posed by non-strictness makes the mental domain ineliminable. However, non-strictness is not an antidote to all mind-body problems. For example, it does not answer the charge that mental properties are epiphenomenal.

It is important to observe that the non-strictness required to make room for irreducible mental properties need not be any sense of full-blown anomalism. Ceteris paribus psychophysical laws will do. Thus it is common to be an 'anomalous monist' about many or all of the special sciences: geology, biology, etc. ${ }^{15}$ 'Mental Events' demonstrated that one could also be an anomalous monist about psychology, and the argument from externalism shows one reason why psychophysical laws are not strict. Had philosophers felt a biology-physics problem in addition to the mind-body problem (as they did in times of vitalism), then anomalous monism about the biological domain would have been seen to contribute towards a solution to that problem too.

\section{SUMMARY}

Davidson claims in 'Could There Be a Science of Rationality?' that externalism entails the anomalousness of the mental. But an attempt to convert these general considerations into a strict argument from reasonable assumptions reaches only a qualified conclusion: there cannot be strict laws of physical to mental causation, in the important class of cases in which (I) the mental entity is extrinsically individuated; and (2) the physical causes are more proximal to the mental event than the contextual or historical factors on which differences in mental content depend. Similarly, there cannot in

15 Davidson has accepted the possibility of anomalous monism about biology, in 'Psychology as Philosophy', in S. Brown (ed.), Philosophy of Psychology (London: Macmillan,

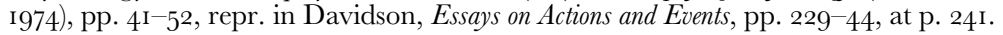


such cases be strict synchronic laws relating the physical to the mental. Furthermore, that result can be consistent with the supervenience of mental properties on physical properties.

Relaxing the assumption of strictness, if the laws of basic physics are exceptionless laws of objective chance, then externalism entails correlative exceptions to probabilistic laws. But the argument-form does not in either case support full-blown anomalousness - it is consistent with the existence of ceteris paribus psychophysical laws. Finally, I claim that even this limited conclusion has great merit: non-strictness demonstrates that there is room for a mental scheme of classification and explanation which does not reduce to basic physics. Thus if externalism is true of some types of mental predicates, and if Davidson is right in characterizing basic physics as a closed and complete scheme of intrinsic predicates applying to everything that exists, then mental talk is ineliminable. ${ }^{16}$

King's College, London

\footnotetext{
${ }_{16}$ Many thanks to Scott Sturgeon for detailed discussion of the issues raised in this paper; and to David Papineau, Jennifer Hornsby, Janne Mantykoski and Scott Brockett for comments on an earlier draft. Thanks are also due to the AHRB for a grant in support of the research reported in this paper.
} 